\title{
Fiber Optic Sensor Array for Perfume Detection Using Neural Network
}

\author{
Bakti Dwi Waluyo $^{1}$, Arwadi Sinuraya ${ }^{2}$, Salman Bintang ${ }^{3}$ \\ \{bakti_dw@unimed.ac.id ${ }^{1}$, arwadisinuraya@unimed.ac.id ${ }^{2}$, salmanbintang@unimed.ac.id $\left.{ }^{3}\right\}$ \\ Universitas Negeri Medan, Indonesia ${ }^{1,2,3}$
}

\begin{abstract}
Optical fiber sensors with a polymer coating instead of the original layer have many advantages to be used as sensors. The advantages of fiber optic sensors are that they have the flexibility of the environment and are free of electromagnetic waves. The working principle of the optical fiber sensor is based on changes in the evanescent field in the boundary area of the core - cladding due to changes in the refractive index when interacting with perfume vapor. Fiber optic sensor testing is done by exposing perfume vapor and letting it interact. During the interaction with perfume vapors, the fiber optic sensor is given a light source from a blue light emitting diode with a wavelength of $450 \mathrm{~nm}$ and as a light sensor is a photodiode that converts light intensity into changes in voltage. The voltage change data is then processed using the artificial neural network method to identify perfume. The experimental results show that the optical fiber sensor array with different polymer cladding and pattern recognition of artificial neural networks can identify perfume.
\end{abstract}

Keywords: fiber optic sensor array, polymer cladding, neural network.

\section{Introduction}

The chemical industry for making cosmetics and perfume products today is growing rapidly. This development resulted in perfume that sold in the market is very vulnerable to falsification. So testing is needed to distinguish the authenticity of perfumes on the market. The tests generally use the sense of smell, this tends to be inaccurate. Because testing using the sense of smell depends on the health condition and mood of the examiner or inspector [1]. Therefore, we need a tool that can detect and distinguish the quality of the scent of perfume.

So far there have been developed sensors based on electronics and optics with chemical sensitive coatings. However light-based sensors are more beneficial because they are not affected by electromagnetic waves, low noise, and can be used for long distances using optical fibers [2].

Optical fiber was originally used as data transmission with the principle of reflection and propagation of light. The reflection and propagation of light in an optical fiber depends on the refractive index between the core and the cladding. Changing or modifying the cladding layer causes a change in the refractive index, causing evanescent waves in the boundary area of the core and cladding [3]. Evanescent waves originating from changes in the refractive index are the main parameters of optical fibers that can be used as sensors [4].

In this study polymer materials have been used as a substitute for original cladding, because polymers are sensitive to chemical vapored so that they can increase sensitivity when interacting with chemical vapored. In principle, polymer cladding will change the refractive 
index in optical fibers when interacting with chemical vapored, resulting in changes in the evanescent field in the core-cladding boundary plane.

Changes in evanescent fields will affect the pattern of light intensity received by the photodiode as a light sensor. Furthermore, the photodiode changes the intensity of light into voltage. The voltage data for each polymer layer will be different when interacting with the gas, so that each type of gas will show different patterns and characteristics. Voltage data from several types of gas are then processed using the Artificial Neural Network (ANN) method, so the results of data processing with ANN are able to identify the types of gas [5].

\section{Optical Fibers}

Optical fibers consist of a core with a refractive index $n_{l}$ surrounded by a cladding with a lower refractive index $n_{2}$. For the conventional fiber (except the photonic crystal fiber), the light transmission in optical fiber is based on the principle of total internal reflection (TIR), which requires the refractive index of the core $\left(n_{1}\right)$ to be higher than that of the cladding $\left(n_{2}\right)$ that is, $n_{1}$ $>n_{2}[4][6]$. As illustrated in Figure 1, important fiber parameters include (a) critical angle $\emptyset \mathrm{c}$, which is defined by the ratio between the cladding and the core refractive indices, as given by

$$
\sin \emptyset_{c}=n_{2} / n_{1}
$$

(b) The acceptance cone angle, $\theta_{\mathrm{i}, \max }$, which depends on the refractive indices of the core, the clad, and the ambient refractive index, $\mathrm{n}_{0}$,

$$
\sin \theta_{i, \max }=\frac{\sqrt{\left(n_{1}^{2}-n_{2}^{2}\right)}}{n_{0}}
$$

And (c) the numerical aperture (NA), which defines the fiber's light collection efficiency and is related to the acceptance cone's angle as:

$$
N A=n_{0} \sin \theta_{i, \max }
$$

All these parameters are critically important when designing the fiber chemical sensors.

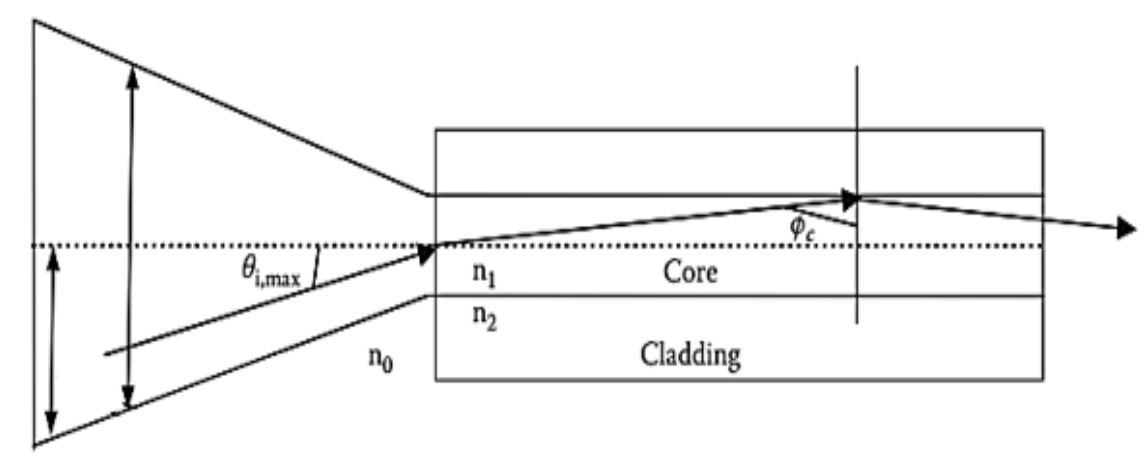

Fig 1. An illustration of light guiding in optical fiber. 


\section{Evanescent Wave}

When the incident light is reflected from an interface at an angle greater than the critical angle, the total internal reflection occurs. However, its intensity does not abruptly decay to zero at the interface and a small portion of light penetrates into the reflecting medium. This penetrated electromagnetic field is called the evanescent wave, as illustrated in Figure 2 [7]. Since the amplitude of evanescent wave decays exponentially with the distance, the penetration depth $(d p)$ is defined as the distance required for the electric field amplitude to fall to 1/e (0.37) of its value at the interface, which is a function of both the wavelength of the light and the angle of incidence, as mathematically given by

$$
d_{p}=\frac{\lambda}{4 \pi\left[n_{1}^{2} \sin ^{2} \theta-n_{2}^{2}\right]^{1 / 2}}
$$

where $\lambda$ is the wavelength of the transmitted light, $\theta$ is the incident angle at the core/cladding interface, and $n_{1}, n_{2}$ are the refractive indices (RI) of the core and cladding, respectively.

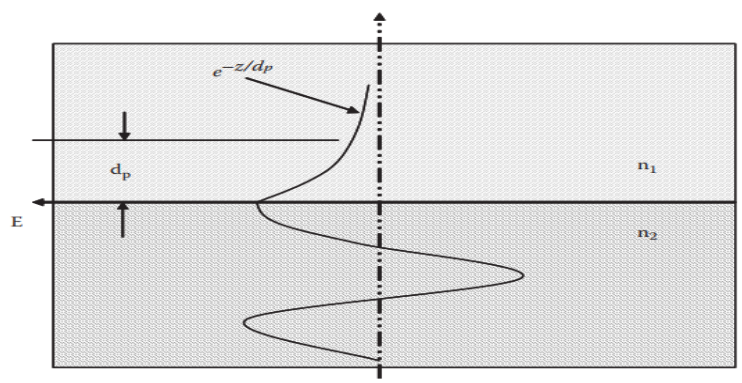

Fig 2. Illustration of exponential decay from evanescent field

\section{Artificial Neural Network}

ANN is a computational model for pattern recognition like a biological neural in the human brain. One of the training methods in ANN is a backpropagation neural network with a multilayer perceptron which is a method of learning with high accuracy. This system must first obtain a training pattern of each type of perfume vapor, and then the training pattern is used as an identification of the type of gas [8]. The backpropagation model will compare the expected outputs and targets, if there are any differences, it will change the (weight) that will be repeated continuously until the target is the same as the output. The model of the backpropagation method can be seen in Figure 3.

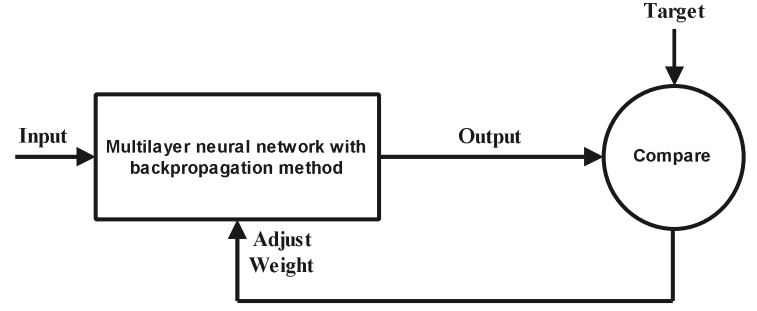

Fig 3. Backpropagation training model. 


\section{Research Method}

The materials used include: acetone as the original cladding peeler, alcohol is used to clean the core surface after the cladding is peeled, and the polymer is used as a substitute for the original cladding. The perfumes that used are Signature Zoom, Ascendant Aqua, and Glacier Rock. The equipment used includes: pliers stripper, Multimode step index plastic optical fiber, Arduino Mega 2560, blue LED with a wavelength of $450 \mathrm{~nm}$, photodiodes, and personal computer.

The optical fiber used is made of Multimode step index plastic type which has a jacket diameter of $2.21 \mathrm{~mm}$, core layer diameter of $0.98 \mathrm{~mm}$, and cladding layer thickness of $125 \mu \mathrm{m}$. The configuration size of the optical fiber pieces can be seen in Figure 4, where the overall length (Lfo) is $25 \mathrm{~cm}$, the length of the right (R) and left (L) is $11 \mathrm{~cm}$. Right (Rx) and left (Lx) lengths of $0.5 \mathrm{~cm}$ each, made by opening the jacket using a stripper. In the middle $(\mathrm{C})$, the original jacket and cladding are removed $2 \mathrm{~cm}$ long. In the center of the optical fiber $(\mathrm{C})$, the original jacket and cladding are removed $2 \mathrm{~cm}$ long. The removal of the original cladding is done by means of a chemical etching method, which is by smearing the core surface with acetone. When the chemical etching process, cladding will experience thickening and will be released from the core slowly. The chemical etching process is carried out at room temperature for approximately two hours. After the original cladding is peeled off, then it is cleaned with alcohol.

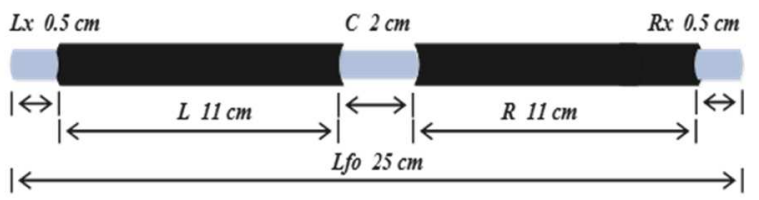

Fig 4. Configuration of optical fiber size

After the optical fiber is cleaned, the next step is to coat the optical fiber core with a polymer. Table 1 is the type of polymer used. The use of different polymers is because each type of polymer has different characteristics, where non-polar polymers tend to interact strongly with non-polar gases and polar polymers tend to interact strongly with polar gases.

Table 1. Types of polymeric materials.

\begin{tabular}{ccc}
\hline Coating & Type Polymer & Constant \\
\hline Layer 1 & Squalane & Non-polar \\
Layer 2 & Apiezone M & Non-Polar \\
Layer 3 & PEG 20M & Polar \\
\hline
\end{tabular}




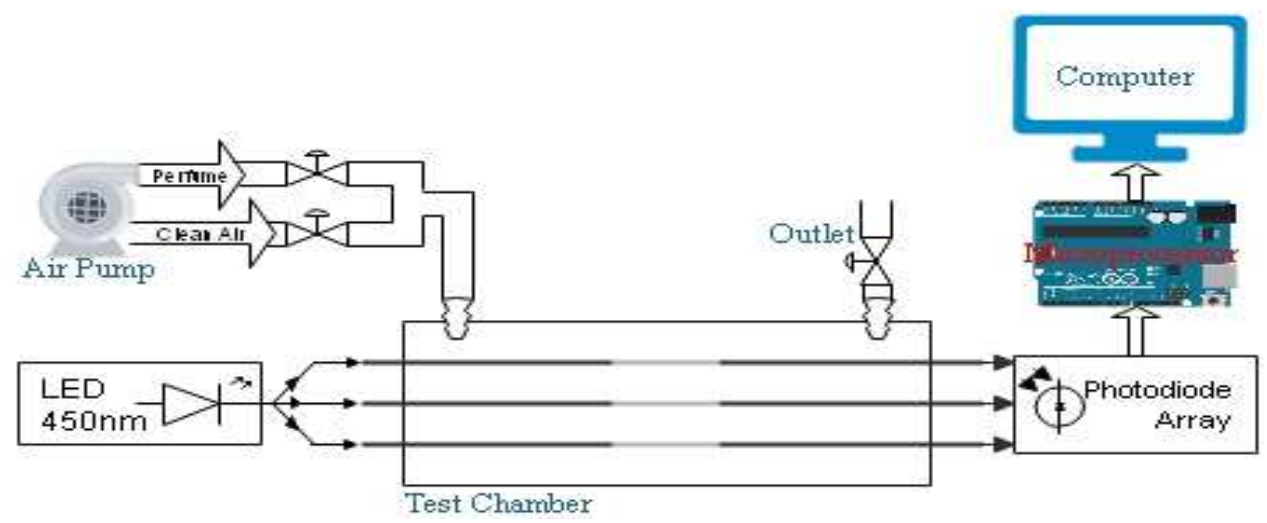

Fig 5. Configuration of optical fiber testing containers.

Illustration of optical fiber testing according to Figure 5, where an air pump with a flow rate of 0.1 Liter Per Minute (LPM) enters silica gel as clean or dry air flowed into the test container. Clean air from silica gel flows into the test container by opening valve 1 and closing valve 2 and valve 3 . After the test container is clean, then drained the gas sample by opening valve 2 and valve 3 , and closing valve 1 .

\section{Result and Discussion}

Data collected for each perfume vapored is carried out four times, where the first test is as learning data on the neural network and three other data are used as an introduction to perfume vapored. The process of data retrieval is done by first flowing clean air into the test container for 30 seconds then perfume vapored is flowed for 140 seconds, then drain clean air again for 330 seconds.

Changes in voltage data for each response of the optical fiber sensor in 160 seconds to 170 seconds can be seen in Table 2. The data analyzed came from the optical fiber response to perfume vapored, which is from 160 to 170 seconds. If the intensity of the light received by the photodiode gets brighter than the graph goes to a negative value (decreases) and if the intensity gets dimmer then the graph tends to be positive (up).

Table 2. Normalization of sensor data

\begin{tabular}{|c|c|c|c|c|c|}
\hline \multirow{2}{*}{ No } & \multirow{2}{*}{ Vapor } & \multirow{2}{*}{ Testing } & \multicolumn{3}{|c|}{ Cladding (Volt) } \\
\hline & & & Squalane & PEG 20M & Apiezone M \\
\hline 1 & \multirow{3}{*}{$\begin{array}{l}\text { Signature } \\
\text { Zoom }\end{array}$} & 1 & 0.181 & 0.239 & 0.159 \\
\hline 2 & & 2 & 0.170 & 0.194 & 0.140 \\
\hline 3 & & 3 & 0.148 & 0.221 & 0.132 \\
\hline \multicolumn{2}{|c|}{ Voltage Average } & & 0.166 & 0.218 & 0.144 \\
\hline 4 & \multirow{3}{*}{$\begin{array}{l}\text { Ascendent } \\
\text { Aqua }\end{array}$} & 1 & 0.175 & 0.133 & 0.113 \\
\hline 5 & & 2 & 0.181 & 0.115 & 0.091 \\
\hline 6 & & 3 & 0.163 & 0.115 & 0.097 \\
\hline \multicolumn{2}{|c|}{ Voltage Average } & & 0.173 & 0.121 & 0.100 \\
\hline 7 & \multirow{3}{*}{$\begin{array}{l}\text { Glacier } \\
\text { Rock }\end{array}$} & 1 & 0.155 & 0.139 & 0.095 \\
\hline 8 & & 2 & 0.154 & 0.137 & 0.096 \\
\hline 9 & & 3 & 0.130 & 0.151 & 0.104 \\
\hline \multicolumn{2}{|c|}{ Voltage Average } & & 0.146 & 0.142 & 0.098 \\
\hline
\end{tabular}


The response of the optical fiber sensor to three different perfume vapors can be seen as follows: Figure 6 is the intensity of the light on the optical fiber sensor when interacting with the signature zoom perfume, Figure 7 is the intensity of the light on the optical fiber sensor when interacting with ascendant aqua perfume, and Figure 8 is the intensity of the light on the optical fiber sensor when interacting with glacier rock perfume.

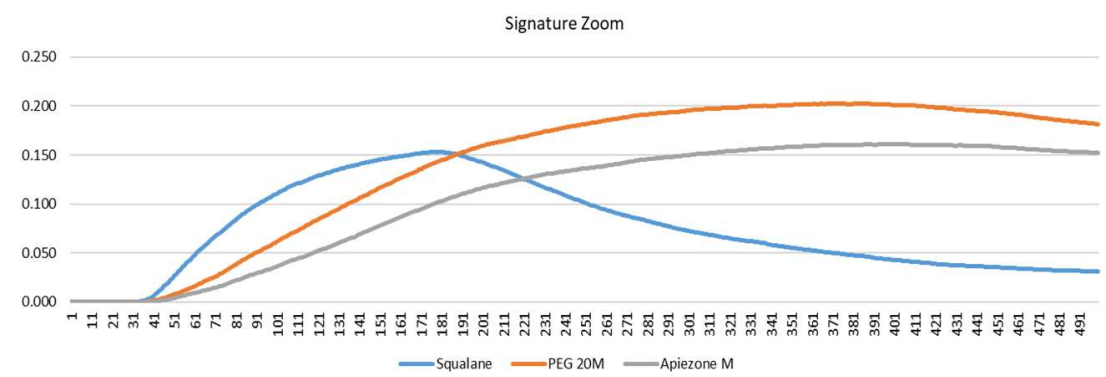

Fig 6. Intensity of optical fiber sensor light to signature zoom perfume vapor. Ascendant Aqua

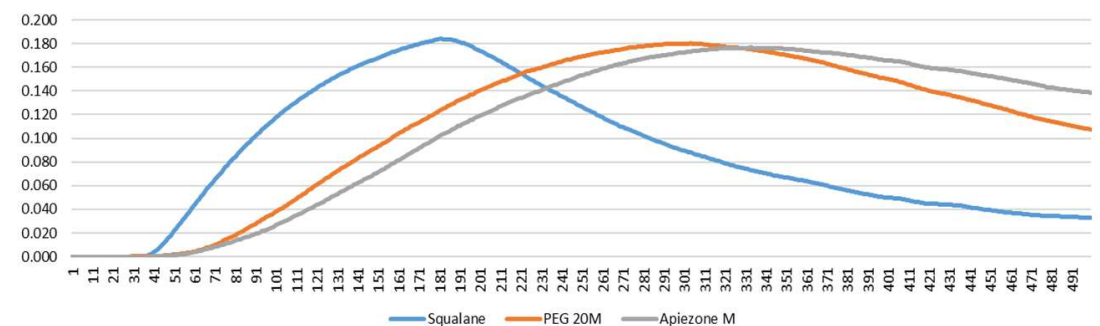

Fig 7. Intensity of optical fiber sensor light to signature ascendant aqua vapor.

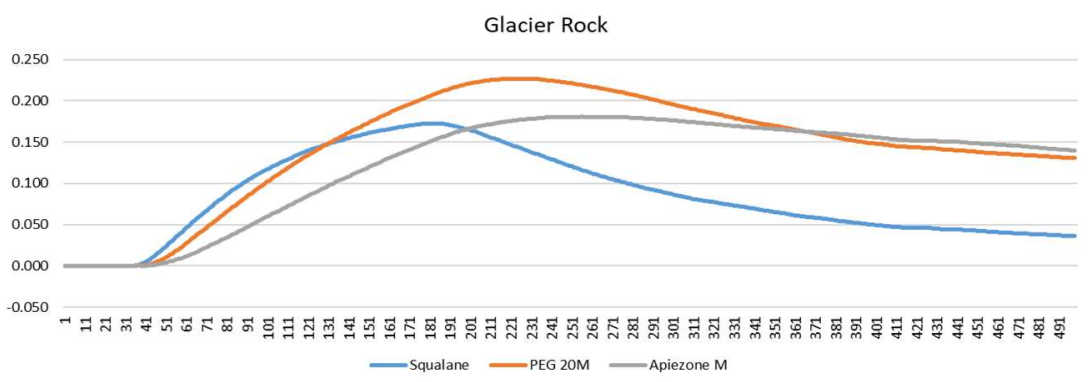

Fig 8. Intensity of optical fiber sensor light to signature glacier rock vapor.

The data in Table 2 are the voltage response of the three polymer layers, so that it can be seen that the polymer layer has a high, medium and low response to the given perfume vapor. Based on the voltage average normalization, it will be seen the response of each polymer layer in each optical fiber, this can be seen in Figure 9. 
The normalized data in Table 2 then becomes a parameter to the learning process in neural networks. This parameter will be used as the next type of perfume vapor testing. Neural network learning is done by setting the desired error value, where the error value is 0.0001 . After the error value is fulfilled, the final weight value is obtained to identify the type of perfume vapored. Data obtained from testing using artificial neural networks are shown in Table 3, from 9 data that has been tested, it can be identified as a whole, so that the success of testing to recognize perfume vapored is $100 \%$.

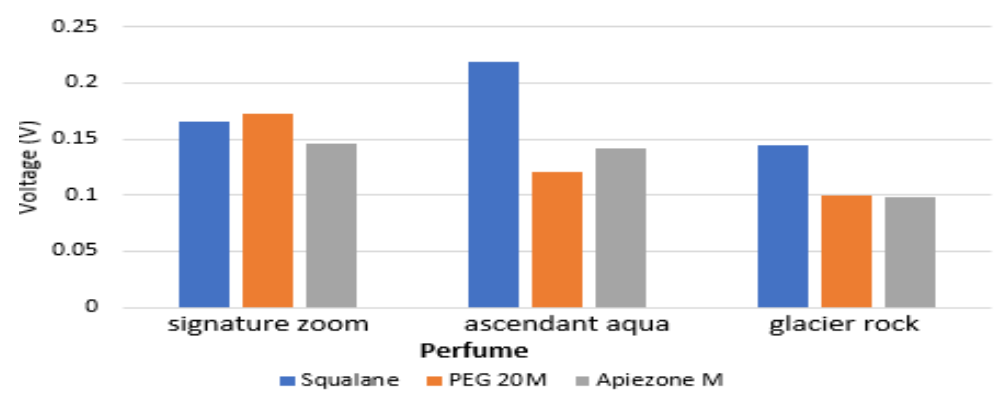

Fig 9. Average voltage normalization of three perfume vapours.

Table 3. Test results with neural network

\begin{tabular}{clccc}
\hline No & Perfum & Testing & Status & (\%) Result \\
\hline 1 & Signature & 1 & match & \\
2 & Zoom & 2 & match & $100 \%$ \\
3 & & 3 & match & \\
4 & Ascendant & 1 & match & \\
5 & 2 & match & $100 \%$ \\
6 & Aqua & 3 & match & \\
7 & & 1 & match & \multirow{2}{*}{ Glacier } \\
8 & Rock & 2 & match & $100 \%$ \\
9 & 3 & match & \\
\hline \multicolumn{5}{c}{ Total } \\
\hline \multicolumn{5}{c}{$100 \%$} \\
\hline
\end{tabular}

\section{Conclusions}

In this research, optical fiber-based gas sensors have been designed and made. This sensor is based on evanescent field changes in the core-cladding boundary area due to changes in the refractive index when interacting with perfume vapored. The chemicals used as polymer cladding are Squalene, Apiezone M, PEG 20M. The test results show that this sensor system is able to distinguish the types of perfume vapored, where there are different responses between perfumes vapored from one another. 


\section{References}

[1] T. Nakamoto, A. Fukuda, and T. Moriizumi. Perfume and flavour identification by odoursensing system using quartz-resonator sensor array and neural-network pattern recognition. Sensors Actuators B. Chem., vol. 10, no. 2, (1993).

[2] W. Cao and Y. Duan. Optical fiber-based evanescent ammonia sensor. Sensors Actuators, B Chem., vol. 110, no. 2, (2005).

[3] V. G. Pahurkar, Y. S. Tamgadge, A. B. Gambhire, and G. G. Muley. Evanescent wave absorption based polyaniline cladding modified fiber optic intrinsic biosensor for glucose sensing application. Meas. J. Int. Meas. Confed., vol. 61, (2015).

[4] F. T. S. Yu, Fiber Optic sensors. (2017).

[5] Q. Zhang, H. Yu, M. Barbiero, B. Wang, and M. Gu. Artificial neural networks enabled by nanophotonics. Light Sci. Appl., vol. 8, no. 1 (2019).

[6] M. Sheeba, M. Rajesh, C. P. G. Vallabhan, V. P. N. Nampoori, and P. Radhakrishnan, Fibre optic sensor for the detection of adulterant traces in coconut oil. Meas. Sci. Technol., vol. 16 , no. 11, (2005).

[7] E. Udd and W. B. Spillman. Fiber Optic Sensors: An Introduction for Engineers and Scientists: Second Edition. (2011).

[8] R. Kumar, R. R. Das, V. N. Mishra, and R. Dwivedi. A neuro-fuzzy classifier-cumquantifier for analysis of alcohols and alcoholic beverages using responses of thick-film tin oxide gas sensor array. IEEE Sens. J., vol. 10, no. 9, (2010). 